\title{
Estudio Estructural de una Medida Breve de Inteligencia Emocional en Adultos: El EQ-i-M20
}

\author{
Structural Study of a Brief Measure of Emotional Intelligence in Adults: The EQ-i- \\ M20
}

\author{
Sergio Alexis Dominguez-Lara ${ }^{1}$, César Merino-Soto ${ }^{2}$ y Andrés Gutiérrez-Torres ${ }^{3}$
}

\begin{abstract}
Resumen
El presente estudio tuvo como objetivo analizar la estructura interna de una medida breve de inteligencia emocional en adultos (EQ-i-M20) en 332 universitarios limeños entre 18 y 56 años (M=23.98, DE=5.497). Luego de evaluar los modelos de medición de cinco factores oblicuos, bifactor y unidimensional, el primero presenta mejor ajuste. Del mismo modo, las correlaciones con criterios externos muestran resultados teóricamente esperados. El análisis de confiabilidad realizado muestra resultados satisfactorios de puntuaciones observadas como variables latentes. Los resultados del estudio confirman que el EQ-i-M20 presenta una estructura de cinco factores bien definidos. Se discuten los resultados y sus implicancias.
\end{abstract}

Palabras clave: inteligencia emocional, análisis factorial confirmatorio, validez, confiabilidad

\begin{abstract}
The aim of this study was to analyse the factorial structure of a brief measure of emotional intelligence in adults (EQ-i-M20) in a sample of 332 college students from Lima City, aged between 18 and 56 years $(\mathrm{M}=23.98, \mathrm{SD}=5.497)$. After assessing three measurement models; a five-factor oblicuous model, bifactor model, and unidimensional model, it is concluded that the oblicuous presents the best model fit. Similarly, correlations with external criteria shown theoretically expected results. Reliability analysis evidences satisfactory levels, both at the observed scores and latent trait. The results obtained in the study confirm that EQ-i-M20 presents a well-defined five-factor structure. Finally, the results and possible consequences are discussed.
\end{abstract}

Keywords: emotional intelligence, confirmatory factorial analysis, validity, reliability

\footnotetext{
${ }^{1}$ Magíster en Psicología Clínica y de la Salud. Docente, Universidad de San Martín de Porres. Instituto de Investigación de Psicología, Universidad de San Martín de Porres, Av. Tomás Marsano 242 (5to piso), Lima 34 - Perú. Tel.: 0051988053909. Correo: sdominguezmpcs@gmail.com

${ }^{2}$ Magíster en Psicología Educativa. Docente, Universidad de San Martín de Porres. Instituto de Investigación de Psicología, Universidad de San Martín de Porres, Av. Tomás Marsano 242 (5to piso), Lima 34 - Perú. Tel.: +515136300 anexos 2186 - 2096. Correo:sikayax@yahoo.com.ar

${ }^{3}$ Magíster en Psicología con mención en intervención psicológica e investigación. Docente, Nacional Mayor de San Marcos. Departamento Académico de Psiquiatría-Facultad de Medicina, Universidad Nacional Mayor de San Marcos, Av. Grau 755 ( $2^{\circ}$ piso), Lima 1 - Perú. Tel.: 0051999933513. Correo: and_gut@hotmail.com

Revista Iberoamericana de Diagnóstico y Evaluación - e Avaliaçã̃o Psicológica. RIDEP · №49 · Vol.4 · 5-21 · 2018 ISSN: $1135-3848$ print /2183-6051online
} 


\section{Introducción}

En la actualidad, parecer ser que la Inteligencia Emocional (IE) es uno de los constructos que aún suscita atención entre los investigadores, debido a su relación con un gran número de variables conductuales y afectivas; por nombrar algunos de estos, los correlatos de IC se han verificado con satisfacción con la vida (Cazan \& Năstasă, 2015), bienestar (Sánchez-Álvarez, Extremera, \& Fernández-Berrocal, 2016), esquemas cognitivos disfuncionales (Dimitriu, \& Negrescu, 2015), autoestima (Abdollahi \& AbuTalib, 2016; Cheung, Cheung, \& Hue, 2015), habilidades sociales (Garaigordobil \& Peña, 2014), conducta prosocial (Martin-Raugh, Kell, \& Motowidlo, 2016), depresión (Lloyd, MalekAhmdi, Barclay, Fernandez, \& Chartrand, 2012; Salguero, Extremera, \& Fernández-Berrocal, 2012), ideación suicida (Suárez, Restrepo, \& Caballero, 2016), burnout (Năstasă \& Fărcaş, 2015; Pishghadam \& Sahebjam, 2012; Torres, \& Cobo, 2017), ansiedad (Nolidin, Downey, Hansen, Schweitzer, \& Stough, 2013), salud física (Mikolajczak \& Van Bellegem, 2017), desempeño laboral (Joseph, Jin, Newman, \& O'Boyle, 2015; Mshellia, Malachy, Sabo, \& Abu-Abdissamad, 2016); por otro lado, también ha sido observado su rol moderador en conductas antisociales y adictivas (Parker, Summerfeldt, Taylor, Kloosterman, \& Keefer, 2013; Schokman et al., 2014). En conjunto, la IE es relevante en la descripción y explicación del proceso de adaptación social de la persona.

Existen dos grandes modelos explicativos de la IE. El primero de ellos la concibe como una parte de la inteligencia social que incluye la capacidad de controlar las emociones propias y las de los demás, discriminar entre ellas y usar esa información para guiar el pensamiento y la conducta (Salovey \& Mayer, 1990). Dentro de esta conceptualización fue incluida la percepción, asimilación, comprensión, y regulación de las propias emociones y de los demás (Mayer \& Salovey, 1997), cada una de las cuales son identificadas como habilidades cognitivas (Mayer, Salovey, \& Caruso, 2002). Los instrumentos utilizados en este enfoque son consideradas medidas de habilidad, debido a que se focalizan en tareas de ejecución cuyas respuestas se comparan posteriormente con criterios objetivos. Estos instrumentos no evalúan las creencias sobre la habilidad, reduciendo así el sesgo de autoevaluación inherente a los autoinformes (Extremera \& Fernández-Berrocal, 2004). El segundo modelo es el propuesto por Bar-On $(1997,2006)$, quien define la IE como un conjunto de competencias, aptitudes y facilitadores emocionales y sociales que determinan la eficacia de las personas para comprenderse y comprender a los demás, para expresarse y relacionarse con otras personas, y para hacer frente a las demandas diarias. Se le conoce además como enfoque mixto, dado que además de aspectos emocionales propiamente dichos, involucra rasgos vinculados con el funcionamiento social, e inclusive el laboral (Extremera, Fernández-Berrocal, Mestre, \& Guil, 2004; Petrides \& Furnham, 2006). Las medidas derivadas de este modelo son las de autoinforme.

Las divergencias conceptuales entre ambos enfoques se ven corroboradas también en sus aspectos empíricos, ya que las puntuaciones de los autoinformes con base en el enfoque mixto se ven influidos en mayor grado por variables de personalidad (Petrides et al., 2016), y en menor medida en las puntuaciones provenientes de medidas de habilidad (Brackett \& Mayer, 2003; Siegling, Saklofske, Vesely, \& Nordstokke, 2012; Webb et al., 2013). Sin embargo, es razonable pensar que ambos aspectos (rasgo y habilidad) coexisten en alguna proporción aun cuando el proceso de construcción de sus indicadores conductuales trate de reducir la covariación entre ambas.

Independientemente de las ventajas y desventajas de ambas aproximaciones, el diseño de una investigación que incorpore la IE como constructo relevante requiere identificar el enfoque que sirve de marco para el uso del constructo en un contexto particular (Extremera et al., 2004; Extremera \& Fernández-Berrocal, 2004). En este caso, en el ámbito universitario es más útil construir un perfil de la IE del estudiante basado en rasgos de personalidad, es decir, el enfoque mixto, pues brinda un panorama general del funcionamiento del individuo, considerando además la evidencia acerca de la relación de la IE con el rendimiento académico (Páez \& Castaño, 2015), en un contexto en que la evidencia 
científica demuestra que hay factores no cognitivos o de habilidad que también explican la varianza del rendimiento (Inzunza et al., 2015; Medrano, Moretti, \& Ortiz, 2015)..

En correspondencia con el enfoque mixto, el instrumento EQ-i (Bar-On, 1997) fue creado con el objetivo de evaluar cinco dimensiones de segundo orden de la IE (Intrapersonal, Interpersonal, Manejo del Estrés, Adaptabilidad, y Ánimo general), mediante 15 diferentes facetas o constructos de primer orden. La estructura interna del EQ-i fue analizada en diversos grupos, como niños y adolescentes (EQ-i: YV; Bar-On \& Parker, 2000; Ferrándiz, Hernández, Bermejo, Ferrando, \& Sáinz, 2012; Sáinz, Ferrándiz, Fernández, \& Ferrando, 2014), y universitarios (López-Zafra, Pulido, \& Berríos, 2014; Parker, Keefer, \& Wood, 2011). Asimismo, algunos estudios evaluaron su vinculación con constructos teóricamente afines y obtuvieron resultados confirmatorios respecto a la teoría de IE (Dawda, \& Hart, 2000; Hemmati, Mills, \& Kroner, 2004; Newsome, Day, \& Catano, 2000). Sin embargo, en estos nuevos contextos de uso, en los que la población es diferente a la de los estudios originales, no se corroboró previamente la estructura interna hipotetizada, y consecuentemente los resultados obtenidos pueden mostrar alguna pérdida de validez interna debido que los constructos pueden no estar adecuadamente representados por sus indicadores esperados.

En el contexto peruano destaca la adaptación realizada del EQ-i: YV (Ugarriza, 2001; Ugarriza, \& Pajares, 2005), sin embargo un estudio metodológico posterior indicó que sus estimaciones de confiabilidad son bastante modestas (Merino, Navarro, \& García, 2014), y limitan seriamente el uso de instrumento en la práctica clínica. De forma similar, existe una traducción del manual técnico de la versión para adultos que aparentemente es de uso extendido en Perú (Abanto, Higueras, \& Cueto, 2000), pero no se cuenta con estudios empíricos apropiados sobre su estructura interna a fin de acreditar su empleo en población general o universitaria peruana. En síntesis, las versiones utilizadas en el contexto peruano carecen de las propiedades psicométricas adecuadas, y se puede afirmar que aún desconoce la generalización de la validez estructural del EQi.

Una revisión de los estudios psicométricos que analizó la estructura interna (Rios \& Wells, 2014) del EQ-i en diferentes versiones, indicó que el método analítico factorial de partida fue el exploratorio (Ferrándiz et al., 2012; López-Zafra et al., 2014; Sáinz et al., 2014; Ugarriza, 2001; Ugarriza \& Pajares, 2005), y con un predominio de la estrategia Little Jiffy (Kaiser, 1960; Componentes principales - valores Eigen > 1 rotación varimax). Los resultados, en términos generales, no reflejaron la estructura pentafactorial original del instrumento, lo cual es una limitación seria a la generalización de la validez estructural del instrumento y de su teoría. Otros estudios partieron desde el enfoque confirmatorio, poniendo a prueba la estructura original del EQ-i. Estos evaluaron el constructo general (inteligencia emocional) a través de un modelamiento jerárquico directo (Stanimirovic \& Hanrahan, 2012) o indirecto (Parker et al., 2011), decantándose en todos los casos por el modelo de factores oblicuos. Finalmente, de forma casi exclusiva se reporta el coeficiente $\alpha$ en casi todas las versiones analizadas, pero sin evaluar los supuestos que harían posible su utilización.

Recientemente, Pérez-Fuentes, Gázquez, Mercader y Molero (2014) derivaron satisfactoriamente una versión de 20 ítems (EQ-iM20) a partir del EQ-i: YV, orientada a la evaluación de adultos mayores que cursaban educación universitaria en España. Quizá es la versión más reducida del EQ-i en idioma español hasta la fecha, la cual da ventajas para su aplicación masiva y posible representación parsimoniosa de sus constructos.. En su estudio, el modelo de cinco factores oblicuos presentó mejor ajuste, incluso por encima del modelo jerárquico indirecto (Canivez, 2016); y las cargas factoriales fueron mayores que .50, lo cual da información sobre la validez convergente de los ítems. Si bien no reportaron los coeficientes de confiabilidad, el cálculo del coeficiente $\alpha$ desde el método de factor común (Dominguez-Lara, 2012) y el coeficiente $\omega$ (McDonald, 1999), presentaron magnitudes aceptables en cada factor: Intrapersonal $(\omega=.806$; $\alpha=.800)$, Interpersonal $(\omega=.691 ; \alpha=.688)$, Manejo del estrés $(\omega=.846 ; \quad \alpha=.842), \quad$ Adaptabilidad 
$(\omega=.718 ; \alpha=.716)$, y Ánimo general $(\omega=.789 ;$ $\alpha=.785)$.

El EQ-i-M20 es útil por su brevedad y la relativa facilidad para incorporarlo en baterías psicológicas de evaluación en el contexto universitario, ya que la versión de 133 ítems disponible en el contexto peruano (Abanto et al., 2000) no cuenta con evidencias de validez para ser aplicada. Pero si las tuviera, su extensión dificultaría su inclusión ya que puede provocar con mayor facilidad cansancio o incluso aburrimiento. De este modo, una herramienta de evaluación de la IE breve y robusta en cuanto a las dimensiones que evalúa podría ser usada con mayor frecuencia por los investigadores, posibilitando así el incremento del conocimiento en torno a la IE y su relación con otros constructos. Con respecto al aspecto metodológico, si bien los procedimientos reportados previamente resultan útiles, estos presentan algunas limitaciones para los objetivos psicométricos. Por ejemplo, el uso recurrente de la combinación Little Jiffy supone un error metodológico grave, puesto que ese método está desaconsejado para los estudios factoriales (Ferrando, \& Anguiano-Carrasco, 2010; LloretSegura, Ferreres-Traver, Hernández-Baeza, \& Tomás-Marco, 2014). Además, utilizar una rotación ortogonal (varimax) es inadecuado porque se conoce que los componentes del EQ-i tienen afinidad teórica, lo que metodológicamente justifica modelar la correlación entre ellas. Por otro lado, exceptuando los estudios que emplean análisis confirmatorios, no se han llevado a cabo análisis que acrediten empíricamente un factor general, lo que conllevaría al uso de una puntuación total. Respecto a ello, un modelo plausible sería el modelo jerárquico directo (bifactor; Canivez, 2016; Reise, 2012), ya que permite estimar de forma simultánea los efectos que proporciona la dimensión general (en este caso, IE) sobre los ítems, y al mismo tiempo revelar los efectos de las dimensiones específicas sobre estos. Finalmente, el uso del coeficiente $\alpha$ no se ha justificado en ningún estudio a través del análisis previo de sus supuestos (tau-equivalencia y ausencia de errores correlacionados), por lo cual los resultados obtenidos podrían estar sesgados.

En este sentido, el presente estudio evalúa las condiciones previas para brindarle mayor sustento a la estimación. Asimismo, considerando que el coeficiente $\alpha$ calcula la confiabilidad de las puntuaciones observadas, se implementarán otros indicadores de confiabilidad como el $\alpha_{\text {ordinal }}$ (Gadermann, Guhn, \& Zumbo, 2008; DominguezLara, 2012) coeficiente $\omega$ (McDonald, 1999) y el coeficiente $H$ (Dominguez-Lara, 2016; Hancock, \& Mueller, 2001), quienes actúan a nivel de variables latentes (constructos). Entonces, los objetivos de la presente investigación fueron a) reportar las evidencias de validez por medio del análisis de la estructura interna del EQ-i-M20 desde la metodología SEM (Structural Equation Modeling); b) de su relación con otros constructos teóricamente relevantes; y c) el análisis de confiabilidad, evaluando sus supuestos estadísticos, y la implementación de coeficientes centrados en las variables latentes. También se reportaron indicadores de confiabilidad de los criterios externos a través del coeficiente $\alpha$ (Cronbach, 1951) y Angoff-Feldt $\left(r_{A F}\right.$; Dominguez-Lara, Merino-Soto, \& Navarro-Loli, 2016; Feldt, \& Charter, 2003).

\section{Método}

\section{Participantes}

La muestra fue conformada por 332 estudiantes universitarios $(76.8 \%$ mujeres $)$ peruanos, con edades entre 18 y 56 años $(M=23.98, D E=5.497), 41.9 \%$ trabajan, y $86.1 \%$ de ellos se encontraba soltero al momento de realizar la encuesta. No se encontraron diferencias significativas en la edad según el sexo $\left(t_{(323)}=0.220, \quad d=0.018\right)$. Provienen de una universidad privada ubicada en Lima Metropolitana, que predominantemente recibe estudiantes de nivel socioeconómico medio.

\section{Instrumentos}

Brief Emotional Intelligence Inventory for Senior Citizens (Pérez et al., 2014; EQ-i-M20). Es un instrumento derivado del EQ-i: YV (BarOn, \& Parker, 2000) mediante un procedimiento analítico factorial en población adultos mayor que cursa estudios universitarios. Consta de 20 ítems en escalamiento Likert de cuatro puntos (Nunca me pasa, A veces me pasa, Casi siempre me pasa, y Siempre me pasa). Evalúa las cinco áreas del 
EQ-i original: Intrapersonal, Interpersonal, Manejo del Estrés, Adaptabilidad, y Ánimo general. El significado de las puntuaciones es directo en todas las subescalas, exceptuando Manejo del Estrés, en el cual una puntuación elevada implica menor manejo del estrés. En su estudio de creación, sus puntuaciones obtuvieron coeficientes de confiabilidad que variaron entre .688 y .842 .

\section{Patient Health Questionnarie-2 (PHQ-2;}

Kroenke, Spitzer, \& Williams, 2003). Compuesto de dos ítems que exploran aspectos emocionales y cognitivos vinculados con la depresión escalados en formato ordinal de respuesta (Para nada, Varios días, Más de la mitad de los días, Casi todos los días) ( $r_{A F}=.732 ; \alpha=.723$, IC 95\%: .667, .771). Las instrucciones de respuesta enfatizan el ánimo depresivo, anhedonia y desesperanza.

Generalized Anxiety Disorder Scale-2 (GAD-2; Kroenke, Spitzer, Williams, Monahan, \& Lowe, 2007). Sus dos ítems evalúan conductas vinculadas con la expresión emocional y cognitiva de la ansiedad generalizada. Están escalados en formato ordinal de respuesta (Para nada, Varios días, Más de la mitad de los días, Casi todos los días), y las instrucciones de respuesta se enfocan en ansiedad, y preocupación constante por diversos problemas. $\left(r_{A F}=.663 ; \alpha=.660\right.$, IC $95 \%$ : $.595, .717)$.

Single-Item Self-Esteem Scale (SISE; Robins, Hendin \& Trzeaniewski, 2001). Evalúa la autoestima global mediante un único ítem (Tengo una alta autoestima), escalado en formato ordinal, ante los que la persona debe expresar su grado de acuerdo (Muy en desacuerdo, En desacuerdo, Entre uno y otro, De acuerdo, y Muy de acuerdo). Cuenta con evidencia sobre su validez convergente y divergente con más de 20 criterios conductuales y de personalidad (Robins, Tracy, Trzesniewski, Potter, \& Gosling, 2001; Robins, Trzesniewski, Tracy, Gosling \& Potter, 2002).

\section{Procedimiento}

Fue realizado un análisis preliminar del contenido del EQ-i-M20, cuya versión inicial fue sometida a opinión de profesionales psicólogos que laboran con estudiantes universitarios, quienes sugirieron cambiar algunas palabras con el objetivo de que el contenido de los ítems refleje el uso cotidiano del lenguaje (Tabla 1). Por ejemplo, en el ítem dos se cambió la palabra furia (Me resulta difícil controlar mi ira [furia]) por cólera, ya que está última es de uso más frecuente. Del mismo modo, el verbo enfadar se cambió por enojar en diversos ítems, por ejemplo en el ítem 12 (Me enfado con facilidad), enfado se cambió por enojo. De este modo, ocho ítems fueron modificados levemente sin alterar su relación con la variable latente predeterminada.

Posteriormente, el EQ-i-M20 se administró en la institución universitaria de los estudiantes, en horario habitual de clase. Los responsables del estudio explicaron la condición de participación voluntaria a los estudiantes, y solo se evaluó a quienes aceptaron. Ninguna de las personas evaluadas recibió compensación económica ni beneficios académicos por su participación.

Antes de realizar el análisis estructural del EQ-i-M20, se realizó un análisis descriptivo y exploratorio para evaluar el comportamiento de los ítems, y determinar el cumplimiento de los supuestos estadísticos a fin de evaluar los potenciales sesgos derivados de ello. Para el análisis de la estructura interna del EQ-i-M20 se usó el análisis factorial confirmatorio desarrollado con programa EQS 6.2 (Bentler \& Wu, 2012) bajo las siguientes condiciones: método de máxima verosimilitud (Beaducel \& Herzberg, 2006); matrices policóricas (Lee, Poon, \& Bentler, 1995) debido a que los ítems son medidas ordinales (Dominguez-Lara, 2014); diversos indices de ajuste como el RMSEA ( $\leq .05)$, CFI $(\geq .95)$, y la prueba general $\chi^{2}$, ajustada para atenuar el efecto de la falta de normalidad de las variables $\left(S B-\chi^{2}\right.$; Satorra \& Bentler, 1994).

Dado que el EQ-i-M20 es una versión reciente, se evaluaron modelos vinculados con sus antecedentes psicométricos encontrados: primero, cinco factores oblicuos $\left(\mathrm{M}_{1}\right)$, pues los estudios antecedentes generalmente lo reportan como un modelo razonable, considerando la afinidad teórica de las subescalas y el planteamiento original del autor. El segundo modelo $\left(\mathrm{M}_{2}\right)$ propuso la configuración bifactor, que especifica adicionalmente un factor general que influye a todos los ítems. De este modo, se pretende identificar el monto de varianza común y especifica 
Tabla 1. Adaptación lingüística del EQ-i-M20

\begin{tabular}{lcc}
\hline $\begin{array}{l}\mathrm{N}^{\circ} \\
\text { ítem }\end{array}$ & Versión original & Versión adaptada \\
\hline 1 & Entiendo bien cómo se sienten las otras personas. & Entiendo cómo se sienten las otras personas. \\
2 & Me resulta difícil controlar mi ira (furia). & Me resulta difícil controlar mi ira (cólera). \\
3 & Me resulta fácil decirle a la gente cómo me siento. & Me resulta fácil decir cómo me siento. \\
12 & Me enfado con facilidad. & Me enojo con facilidad. \\
13 & Me gusta hacer cosas para los demás. & Me gusta hacer cosas para ayudar a los demás. \\
16 & Es fácil para mí decirle a la gente lo que siento. & Es fácil para mí decir lo que siento. \\
18 & Cuando me enfado, actúo sin pensar. & Cuando me molesto, actúo sin pensar. \\
20 & Me gusta cómo me veo. & Me gusta cómo me veo en general. \\
\hline
\end{tabular}

en cada ítem, y valorar la viabilidad de un factor general y los cinco factores específicos del EQ-iM20, dado que en la literatura se aboga por un factor general. Para valorar el modelo se usó, además de los índices de ajuste el $\omega_{h}$ (Zinbarg, Yovel, Revelle, \& McDonald, 2006), el cual hace referencia al monto de varianza total que puede ser atribuida al factor general, y el $E C V$ (Explained Common Variance; Reise, Scheines, Widaman, \& Haviland, 2013) que se interpreta como el monto de varianza común que se debe al factor general. Un ECV mayor que .60 es indicador de que hay poca varianza común entre factores más allá que la del factor general; y el PUC (Percentage of Uncontaminated Correlations; Reise et al., 2013) que brinda información sobre el porcentaje de correlaciones no contaminadas por la multidimensionalidad (Rodriguez, Reise, \& Haviland, 2015). Estos procedimientos son necesarios porque permiten evaluar el impacto que tiene el factor general con respecto a los factores específicos sobre los ítems. También se evaluó el modelo unidimensional $\left(\mathrm{M}_{3}\right)$, que propone una sola dimensión subyacente de los ítems.

Una vez identificado el modelo con mejor ajuste estadístico y con más racionalidad teórica, se evaluaron los modelos de medición congenérico, tau-equivalente y paralelo en cada subescala, comparando el ajuste de los modelos con su versión menos restricta a través de las variaciones en el CFI (Cheung, \& Rensvold, 2002). Posteriormente, usando el coeficiente por rangos de Spearman, se asociaron las puntuaciones de los componentes del EQ-i-M20 con medidas de ansiedad, depresión y autoestima (Hemmati et al., 2004), esperando correlaciones inversas entre las subescalas Intrapersonal, Interpersonal, Adaptabilidad, y Ánimo general con ansiedad y depresión, y correlaciones positivas con autoestima; por otro lado, se espera que Manejo del estrés correlacione de forma directa con ansiedad y depresión y negativamente con autoestima (Dawda, \& Hart, 2000). Fueron utilizadas tres medidas breves para evaluar dichos criterios, aunque existen estudios que acreditan sus bondades psicométricas a nivel internacional. Esto se realizó con el objetivo de maximizar la tasa de respuestas válidas entre los encuestados, ya que instrumentos extensos pueden disminuir la intención de colaborar en la evaluación. Además, existe evidencia de instrumentos que evalúan constructos multidimensionales con pocos ítems por dimensión ( $1-3$ reactivos), tanto creados como validados en el ámbito peruano, que han presentado evidencia psicométrica favorable (Dominguez-Lara, en prensa; Dominguez-Lara \& Merino-Soto, 2015a, en prensa; Merino-Soto, Dominguez-Lara, \& Fernandez-Arata, 2017).

Finalmente, en cuanto al análisis de la confiabilidad, se analizaron los supuestos para el uso del coeficiente $\alpha$. Luego de ello, se reportó tanto la confiabilidad de las puntuaciones mediante el coeficiente $\alpha$ con intervalos de confianza (IC; Dominguez-Lara, \& Merino-Soto, $2015 b)$, como el $\alpha_{\text {ordinal, }}$ coeficiente $\omega$ y el coeficiente $H$. Dado que el análisis se focalizó a nivel de variables latentes, la corrección del coeficiente $\alpha$ por errores correlacionados (Pascual-Ferrá \& Beatty, 2015) se realizó usando el promedio de las correlaciones policóricas interitem y el $\alpha_{\text {ordinal }}$ Por su parte, el coeficiente $\omega$ también fue corregido por errores correlacionados (Raykov, 2001). 


\section{Resultados}

\section{Análisis descriptivo y exploración inicial de datos}

De acuerdo con los resultados, la mayor parte de los ítems presentan índices estandarizados de asimetría (Standardized Skew Index; SSI; Malgady, 2007) de magnitud moderada ( $.25 \leq$ SSI $\leq .50$ ); asimismo, algunos ítems presentan el efecto de techo o piso (Terwee et al., 2007), es decir, que más del $15 \%$ de los evaluados obtienen la puntuación máxima o mínima posible en una pregunta.

\section{Análisis de estructura interna}

En primera instancia se evaluó el ajuste del modelo oblicuo de cinco factores $\left(\mathrm{M}_{1}\right)$, presentando índices de ajuste adecuados: SB$\chi_{(160)}^{2}=225.863(p<.01)$, CFI $=.984$, RMSEA $=.035$ (IC 90\%=.024, .045), SRMR=.070. Se destaca la presencia de correlaciones significativas entre factores, pero sin llegar a la multicolinealidad $\left(\varphi_{\mathrm{ij}} \leq .70\right.$, ver Tabla 2$)$. Para verificar esto, se obtuvieron coeficientes de configuración y los coeficientes de estructura (Graham, Guthrie, \&
Thompson, 2003; Thompson, 1997) y un índice de simplicidad factorial (ISF; Fleming, \& Merino, 2005; Navarro-Loli, Merino-Soto, DominguezLara, \& Fleming, 2016), los cuales no sugirieron una estructura general redundante o con exceso de complejidad factorial.

Se analizó el modelo $\mathrm{M}_{2}$, pero debido a presencia de casos Heywood tuvo que realizarse con matrices de covarianzas. Respecto a los índices de ajuste, estos disminuyeron respecto al anterior: SB- $\chi_{(150)}^{2}=219.554(p<.001)$; CFI $=.966$; RMSEA (IC 90\%)=.037 (.026, .048); $\mathrm{SRMR}=.052$. Aunque sus diferencias con $\mathrm{M}_{1}$ parecen no ser significativas en cuanto a las magnitudes de sus índices de ajuste, el objetivo de $\mathrm{M}_{2}$ (bifactor) es ver el grado de influencia del factor general sobre los específicos. De este modo, un análisis descriptivo de los coeficientes de configuración evidencia que, en promedio, los pertenecientes al factor general $\left(\lambda_{\text {promedio }}=.364\right)$ son menores que los pertenecientes a los factores Intrapersonal $\quad\left(\lambda_{\text {promedio }}=.617\right), \quad$ Interpersonal $\left(\lambda_{\text {promedio }}=.541\right)$, Manejo del estrés $\left(\lambda_{\text {promedio }}=.652\right)$, Adaptabilidad $\left(\lambda_{\text {promedio }}=.525\right)$, y Estado de ánimo $\left(\lambda_{\text {promedio }}=.543\right)$. Del mismo modo, el $\omega_{\mathrm{h}}$ (Zinbarg

Tabla 2. Estadísticos descriptivos de los ítems del EQ-i-M20

\begin{tabular}{lccccccc}
\hline & $M$ & $D E$ & $g_{1}$ & $S S I$ & $g_{2}$ & \%Mín & \%Máx \\
\hline Ítem 1 & 2.98 & .668 & -.156 & .175 & -.219 & .9 & 20.2 \\
Ítem 2 & 1.98 & .755 & .702 & .616 & .625 & 24.4 & 4.8 \\
Ítem 3 & 2.58 & .860 & -.005 & .003 & -.658 & 9.6 & 15.1 \\
Ítem 4 & 3.05 & .758 & -.326 & .284 & -.523 & 1.8 & 29.2 \\
Ítem 5 & 2.82 & .720 & .047 & .045 & -.606 & 1.5 & 16.9 \\
Ítem 6 & 2.95 & .748 & -.261 & .233 & -.360 & 2.4 & 22.9 \\
Ítem 7 & 2.68 & .852 & -.065 & .045 & -.672 & 7.5 & 17.8 \\
Ítem 8 & 2.05 & .787 & .774 & .625 & .615 & 21.7 & 6.9 \\
Ítem 9 & 2.63 & .733 & -.074 & .069 & -.268 & 5.1 & 9.9 \\
Ítem 10 & 2.65 & .854 & -.003 & .002 & -.699 & 7.5 & 17.5 \\
Ítem 11 & 2.70 & .774 & -.083 & .069 & -.416 & 5.1 & 14.2 \\
Ítem 12 & 2.08 & .809 & .642 & .490 & .199 & 22.0 & 6.9 \\
Ítem 13 & 3.24 & .747 & -.512 & .459 & -.754 & .6 & 42.2 \\
Ítem 14 & 3.01 & .729 & -.207 & .195 & -.577 & 1.2 & 25.9 \\
Ítem 15 & 3.22 & .757 & -.522 & .455 & -.671 & .9 & 41.3 \\
Ítem 16 & 2.73 & .840 & .085 & .060 & -.858 & 4.5 & 20.8 \\
Ítem 17 & 3.21 & .799 & -.612 & .479 & -.553 & 1.8 & 42.8 \\
Ítem 18 & 2.17 & .830 & .512 & .372 & -.123 & 19.6 & 7.8 \\
Ítem 19 & 2.80 & .771 & .040 & .034 & -.735 & 2.4 & 19.3 \\
Ítem 20 & 3.23 & .761 & -.548 & .473 & -.678 & .9 & 42.5 \\
\hline
\end{tabular}

Nota. $\mathrm{N}=332 ; M$ : media aritmética. $D E$ : desviación estándar; $g_{1}$ : asimetría de Fisher; $g_{2}$ : curtosis de Fisher. SSI: Índice estandarizado de Asimetría; \%Min: porcentaje de personas con la puntuación mínima; \%Máx: porcentaje de personas con la puntuación máxima 
Tabla 3. Parámetros de los ítems, y confiabilidad en EQ-i-M20: modelo oblicuo, bifactor y unifactorial

\begin{tabular}{|c|c|c|c|c|c|c|c|c|c|}
\hline & \multicolumn{5}{|c|}{ Cinco factores oblicuos } & & \multicolumn{2}{|c|}{ Bifactor } & \multirow{2}{*}{$\begin{array}{c}\text { Unifactorial } \\
\mathrm{F}_{1}\end{array}$} \\
\hline & $\mathrm{F}_{1}$ & $\mathrm{~F}_{2}$ & $\mathrm{~F}_{3}$ & $\mathrm{~F}_{4}$ & $\mathrm{~F}_{5}$ & ISF & $\mathrm{F}_{\mathrm{E}}$ & $\mathrm{F}_{\mathrm{G}}$ & \\
\hline Ítem 3 & .653 & .179 & -.027 & .364 & .326 & .542 & .504 & .337 & .466 \\
\hline Ítem 7 & .862 & .236 & -.035 & .481 & .43 & .542 & .727 & .424 & .565 \\
\hline Ítem 10 & .879 & .241 & -.036 & .490 & .439 & .541 & .670 & .496 & .604 \\
\hline Ítem 16 & .823 & .226 & -.034 & .459 & .411 & .541 & .565 & .516 & .647 \\
\hline Ítem 1 & .215 & .783 & -.071 & .450 & .250 & .602 & .612 & .352 & .371 \\
\hline Ítem 5 & .227 & .828 & -.075 & .476 & .264 & .602 & .770 & .359 & .355 \\
\hline Ítem 13 & .119 & .435 & -.040 & .250 & .139 & .602 & .456 & .124 & .443 \\
\hline Ítem 19 & .133 & .486 & -.044 & .279 & .155 & .602 & .326 & .272 & .240 \\
\hline Ítem 2 & -.030 & -.067 & .737 & -.120 & -.251 & .839 & .662 & -.177 & -.229 \\
\hline Ítem 8 & -.031 & -.069 & .760 & -.124 & -.258 & .840 & .704 & -.109 & -.204 \\
\hline Ítem 12 & -.033 & -.074 & .815 & -.133 & -.277 & .840 & .709 & -.202 & -.278 \\
\hline Ítem 18 & -.025 & -.055 & .604 & -.098 & -.205 & .840 & .532 & -.186 & -.251 \\
\hline Ítem 6 & .312 & .322 & -.091 & .560 & .328 & .399 & .506 & .118 & .510 \\
\hline Ítem 9 & .378 & .389 & -.110 & .677 & .397 & .399 & .887 & .461 & .469 \\
\hline Ítem 11 & .456 & .470 & -.133 & .817 & .479 & .399 & .655 & .274 & .609 \\
\hline Ítem 14 & .368 & .380 & -.108 & .660 & .387 & .399 & .050 & .670 & .638 \\
\hline Ítem 4 & .379 & .242 & -.258 & .445 & .760 & .458 & .334 & .665 & .776 \\
\hline Ítem 15 & .442 & 283 & -.301 & .519 & .886 & .458 & .623 & .559 & .776 \\
\hline Ítem 17 & .417 & .266 & -.284 & .489 & .835 & .458 & .647 & .459 & .722 \\
\hline Ítem 20 & .414 & .265 & -.282 & .486 & .830 & .458 & .569 & .517 & .750 \\
\hline F1 & 1 & & & & & & & & \\
\hline $\mathrm{F} 2$ & .274 & 1 & & & & & & & \\
\hline F3 & -.041 & -.091 & 1 & & & & & & \\
\hline $\mathrm{F} 4$ & .558 & .575 & -.163 & 1 & & & & & \\
\hline F5 & .499 & .319 & -.34 & .586 & 1 & & & & \\
\hline AVE & .655 & .431 & .537 & .469 & .687 & & & & \\
\hline$\omega$ & .882 & .738 & .821 & .776 & .898 & & - & - & .816 \\
\hline$\omega_{\mathrm{h}}$ & .560 & .565 & .722 & .518 & .426 & & - & .493 & \\
\hline$H$ & .901 & .812 & .836 & .803 & .905 & & - & - & .911 \\
\hline$H_{\text {bifactor }}$ & .733 & .709 & .761 & .827 & .662 & & - & .822 & - \\
\hline$\alpha$ & .843 & .653 & .766 & .719 & .851 & & - & - & .798 \\
\hline IC95\% Inf. & .809 & .587 & .718 & .663 & .818 & & - & - & .755 \\
\hline IC95\% Sup. & .872 & .711 & .807 & .767 & .878 & & - & - & .834 \\
\hline$\alpha_{\text {ordinal }}$ & .879 & .719 & .818 & .771 & .897 & & - & - & - \\
\hline$r_{\mathrm{ij}}$ & .646 & .385 & .531 & .452 & .680 & & - & - & - \\
\hline$\alpha_{\text {ordinal.corregido }}$ & .854 & .476 & .743 & .753 & .753 & & - & - & - \\
\hline$\omega_{\text {corregido }}$ & .862 & .669 & .787 & .763 & .844 & & - & - & - \\
\hline
\end{tabular}

Nota. $\mathrm{n}=332 ; \mathrm{F}_{1}$ : Intrapersonal; $\mathrm{F}_{2}$ : Interpersonal; $\mathrm{F}_{3}$ : Manejo del estrés; $\mathrm{F}_{4}$ : Adaptabilidad; $\mathrm{F}_{5}$ : Estado de ánimo; ISF: Índice de simplicidad factorial; $\mathrm{F}_{\mathrm{E}}$ : factor específico; $\mathrm{F}_{\mathrm{G}}$ : Factor general; en negrita: Coeficientes de configuración; en itálica: Coeficientes de estructura; AVE: Varianza promedio extraída; $\omega$ : Coeficiente omega; $\omega_{\mathrm{h}}$ : Coeficiente omega jerárquico; $H$ : coeficiente $H ; r_{\mathrm{ij}}$ : promedio de correlaciones policóricas inter-item; $\alpha_{\text {ordinal.corregido }} \mathrm{y} \omega_{\text {corregido }}$ : coeficientes corregidos por errores correlacionados.

et al., 2006) del factor general es menor al de los factores específicos, y otros índices adicionales como $\mathrm{ECV}=.309$, $\mathrm{PUC}=.842$, y coeficientes $\mathrm{H}>.70$ para cada factor específico, indican que los factores específicos son robustos (Rodriguez et al., 2015). Entonces, la solución factorial que puede representar mejor a los datos es la de cinco factores oblicuos. Finalmente, se evalúo el ajuste de un modelo unidimensional $\left(\mathrm{M}_{3}\right)$, esta vez con matrices policóricas, obteniéndose índices de ajuste bastante bajos e insuficientes para sustentar la presencia de un único factor: SB$\chi_{(170)}^{2}=1213.561$ ( $\left.p<.01\right)$; CFI=.753; RMSEA (IC $90 \%)=.136(.129, .143) ; \mathrm{SRMR}=.136$.

\section{Evidencias externas de validez}

A partir de los resultados expuestos en la Tabla 4, se aprecia un patrón correlacional coherente con los antecedentes teóricos de la IE. Las correlaciones son estadísticamente significativas, muchas de ellas de magnitud moderada (e.g., depresión y componente estado de ánimo), y otras de ellas de baja magnitud (e.g., depresión y componente interpersonal). 
Tabla 4. Análisis correlacional de las dimensiones del EQ-i-M20 con criterios externos

\begin{tabular}{cccccc}
\hline & $\mathrm{F} 1$ & $\mathrm{~F} 2$ & $\mathrm{~F} 3$ & $\mathrm{~F} 4$ & $\mathrm{~F} 5$ \\
\hline Ansiedad & $-.142 *$ & $-.120 *$ & $.295 * *$ & $-.230 * *$ & $-.260 * *$ \\
Depresión & $-.230 * *$ & $-.112 *$ & $.311 * *$ & $-.149 * *$ & $-.369 * *$ \\
Autoestima & $.197 * *$ & $.147 * *$ & $-.202 * *$ & $.299 * *$ & $.526 * *$
\end{tabular}

Nota. $n=332$; F1: Intrapersonal; F2: Interpersonal; F3: Manejo del estrés; F4: Adaptabilidad; F5: Estado de ánimo; $* p<.05 ; * * p<.01$

Tabla 5. Evaluación de modelos de medición congenérico y tau-equivalente en el EQ-i-M20

\begin{tabular}{|c|c|c|c|c|c|}
\hline Modelo de medición & $\mathrm{SB}-\chi^{2}(p)$ & CFI & $\Delta_{\mathrm{CFI}}$ & $\begin{array}{l}\text { RMSEA } \\
\text { (IC 90\%) }\end{array}$ & SRMR \\
\hline \multicolumn{6}{|l|}{ F1 } \\
\hline Congenérico & $\begin{array}{c}4.155 \\
(p=.125)\end{array}$ & .998 & .016 & $\begin{array}{c}.057 \\
(.000, .136)\end{array}$ & .021 \\
\hline Tau-equivalente & $\begin{array}{c}20.894 \\
(p<.001)\end{array}$ & .982 & & $\begin{array}{c}.098 \\
(.057, .143)\end{array}$ & .066 \\
\hline \multicolumn{6}{|l|}{$\mathrm{F} 2$} \\
\hline Congenérico & $\begin{array}{c}3.912 \\
(p=.141)\end{array}$ & .992 & .118 & $\begin{array}{c}.054 \\
(.000, .133)\end{array}$ & .035 \\
\hline Tau-equivalente & $\begin{array}{c}33.405 \\
(p<.001)\end{array}$ & .874 & & $\begin{array}{c}.131 \\
(.091, .175)\end{array}$ & .105 \\
\hline \multicolumn{6}{|l|}{ F3 } \\
\hline Congenérico & $\begin{array}{c}5.478 \\
(p=.065)\end{array}$ & .992 & .012 & $\begin{array}{c}.072 \\
(.000, .148)\end{array}$ & .033 \\
\hline Tau-equivalente & $\begin{array}{l}13.782 \\
(p<.05)\end{array}$ & .980 & & $\begin{array}{c}.073 \\
(.028, .120)\end{array}$ & .059 \\
\hline \multicolumn{6}{|l|}{$\mathrm{F} 4$} \\
\hline Congenérico & $\begin{array}{c}.952 \\
(p=.621)\end{array}$ & 1.000 & .033 & $\begin{array}{c}.000 \\
(.000, .087)\end{array}$ & .014 \\
\hline Tau-equivalente & $\begin{array}{l}16.386 \\
(p<.01)\end{array}$ & .967 & & $\begin{array}{c}.083 \\
(.040, .129)\end{array}$ & .070 \\
\hline \multicolumn{6}{|l|}{ F5 } \\
\hline Congenérico & $\begin{array}{c}10.056 \\
(p<.001)\end{array}$ & .993 & .007 & $\begin{array}{c}.110 \\
(.050, .182)\end{array}$ & .030 \\
\hline Tau-equivalente & $\begin{array}{l}21.650 \\
(p<.01)\end{array}$ & .986 & & $\begin{array}{c}.100 \\
(.059, .145)\end{array}$ & .056 \\
\hline
\end{tabular}

\section{Evidencias de Confiabilidad}

Análisis de modelos de medición. Este procedimiento se realizó independientemente con cada factor ya que uno de los requisitos para usar el coeficiente $\alpha$ es que la medida sea unidimensional (Meyer, 2010). Fue evaluado inicialmente el modelo congenérico, para posteriormente forzar la igualdad de coeficientes de configuración y compararlo con el modelo previo mediante las variaciones en el CFI ( $\Delta_{\mathrm{CFI}} \leq .01$; Cheung \& Rensvold, 2002). La Tabla 5 resume todos los resultados.
Posteriormente, a través de los Índices modificadores de Lagrange (IL; Sörbom, 1989) se analizó la pertinencia de modelar errores correlacionados en función de su papel en el incremento del ajuste con cada uno de los factores evaluados, y los IL sugirieron la correlación entre algunos residuales dentro de cada factor. Por razones de identificación del modelo (Brown, 2006), solo pudo modelarse la correlación de un par de errores correlacionados.

En el factor Intrapersonal, la correlación entre los residuales $(\phi)$ de los ítems 3 (Me resulta fácil 
decir cómo me siento) y 16 (Es fácil para mí decir lo que siento) fue de .139 ( $p<.05)$. Asimismo, en el factor Interpersonal la correlación de residuales de los reactivos 1 (Entiendo cómo se sienten las otras personas) y 5 (Sé cómo se sienten las otras personas) fue $.451 \quad(p<.05)$. Por su parte en el factor Manejo del estrés se encontró una correlación de .226 entre los residuales de los ítem 2 (Me resulta difícil controlar mi ira [cólera]) y 18 (Cuando me molesto, actúo sin pensar). A su vez, en el factor Adaptabilidad se encontró una correlación de $.082(p>.05)$ entre los residuales de los ítems 11 (Cuando quiero puedo encontrar muchas formas de contestar a una pregunta difícil) y 9 (Ante preguntas difíciles, puedo dar buenas respuestas). Finalmente, en el factor Estado de ánimo, la correlación de residuales de los ítems 17 (Soy feliz con el tipo de persona que soy) y 20 (Me gusta cómo me veo en general), fue de .384 ( $p<.05)$. Se modelaron estos residuales correlacionados no solo por la evidencia estadística obtenida, sino también debido al análisis conceptual que verificó su coherencia teórica.

Posteriormente, con esos datos se corrigieron los coeficientes de confiabilidad ya que debido a la presencia de errores correlacionados los coeficientes de confiabilidad se incrementan de forma espuria (Dunn, Baguley, \& Brunsden, 2014). Ese procedimiento de corrección se realizó a nivel de los coeficientes $\alpha_{\text {ordinal }}$ y $\omega$ (Tabla 2).

Estimaciones. La confiabilidad fue estimada tanto en variables latentes como de puntuaciones observadas (Tabla 2). El $\alpha_{\text {ordinal }}$ alcanzó niveles adecuados (Gadermann et al., 2012) antes y después de la corrección por errores correlacionados, así como los coeficientes $\omega$ y $H$ (>.80), lo cual indica buena confiabilidad del constructo (Raykov \& Hancock, 2005). El coeficiente $\alpha$ calculado presentó de una magnitud adecuada (Merino et al., 2014) en cada subescala analizada. Un indicador de consistencia interna adicional es la correlación interitem promedio $\left(r_{\mathrm{ij}}\right.$; Cronbach, 1951), la cual se realizó en este estudio a partir de las correlaciones policóricas, aceptándose como adecuados valores entre $40 \mathrm{y}$ .50 (Clark \& Watson, 1995), aunque el componente Interpersonal no alcanza esas magntudes.

\section{Discusión}

El objetivo principal del presente trabajo fue obtener evidencias de validez estructural y confiabilidad por consistencia interna del EQ-iM20 para su uso en el contexto universitario. El modelo que recibió mayor respaldo empírico fue el de cinco factores oblicuos, lo cual coincide con la literatura previa (Fuentes et al., 2014; Parker et al., 2011; Stanimirovic \& Hanrahan, 2012). No obstante, quizá el hallazgo más importante fue que el modelo bifactor no recibió soporte empírico, lo que implica que el uso de una puntuación total no estaría justificado desde nuestro datos, al menos en esta muestra y lo que coincide a su vez con la evidencia preliminar (Fuentes et al., 2014; Parker et al., 2011; Stanimirovic \& Hanrahan, 2012). Esto indica que en el contexto de evaluación psicológica, es recomendable interpretar los cinco factores que evalúa el EQ-i-M20, pero sin obtener una puntuación total.

Dentro de cada una de las subescalas del EQi-M20, los coeficientes de configuración presentaron magnitudes elevadas que reflejan la validez convergente a nivel de ítem, y las correlaciones interfactoriales van de acuerdo con el modelo teórico original. Asimismo, no existe evidencia de superposición empírica de factores que sugiera la presencia de un factor general, lo cual va de acuerdo con los hallazgos preliminares. No obstante, más adelante se observó dependencia local entre algunos ítems (Reise, Waller, \& Comrey, 2000), fundamentalmente con respecto al fraseo, lo que debe estudiarse a profundidad en otras investigaciones. Es posible que esto sea producido por aspectos idiosincrásicos propios a la muestra de participantes, pero un estudio que replique estos residuales correlacionados podrá respaldar su generalización.

Por otro lado, las correlaciones observadas con los criterios externos, si bien son de baja magnitud, se muestran según lo teóricamente esperado. Las subescalas que presentan un patrón correlacional de mayor magnitud con criterios externos son Manejo del Estrés, Estado de ánimo, y Adaptabilidad, lo que coincide con lo reportado previamente con relación a la asociación directa de la IE con la autoestima y síntomas de distrés emocional como la ansiedad y depresión (Abdollahi \& Abu-Talib, 2016; Bar-On, 2005; 
Cheung, Cheung, \& Hue, 2015; Dawda \& Hart, 2000; Hemmati et al., 2004; Lloyd et al., 2012; Newsome, 2000; Nolidin et al., 2013; Salguero et al., 2012). Por otro lado, a diferencia de los estudios previos, las subescalas menos asociadas con los criterios externos fueron Intrapersonal e Interpersonal, quizá por la amplitud de sus enunciados y el carácter específico de las medidas de ansiedad, depresión, y autoestima; también pudo ocurrir que los instrumentos usados como criterios externos solo contaron con uno o dos ítems, lo que le quita potencia estadística además de capitalizar mayor error de medición que el permitido, lo que redunda en indicadores de confiabilidad relativamente bajos, por lo que las interpretaciones deben realizarse con cautela. Asimismo, el efecto suelo y techo de las medidas de ansiedad y depresión atenúa las correlaciones por exceso de asimetría.

Finalmente, el reporte de confiabilidad de las puntuaciones y de las variables latentes brindó indicadores favorables, aunque con cierto grado de heterogeneidad entre los componentes del EQi-M20. Esto es un aspecto a tomar en cuenta, ya que al no cumplirse algunos supuestos para el uso del coeficiente $\alpha$ y encontrarse errores correlacionados, la estimación de la consistencia interna por medio de este coeficiente subestimaría la magnitud de este parámetro. Una consecuencia de este problema es que la puntuación parecería incluir espuriamente más error de medición, y también más amplitud del error estándar de medición. Estos hallazgos tienen implicancias teóricas y metodológicas. Respecto a lo primero, en sentido estricto no podría hablarse de un constructo general (Inteligencia Emocional), sino de un conjunto de rasgos específicos que lo configuran (Petrides, \& Furnham 2006). Por lo tanto, operacionalmente el uso de puntuaciones independientes para describir en un sujeto los componentes del IE tiene respaldo, y no una puntuación global. En tal sentido, cobran relevancia los hallazgos que involucraban de forma significativa las puntuaciones los autoinformes basados en el modelo mixto con variables de personalidad (Siegling et al., 2012; Webb et al., 2013).

Desde lo metodológico, debe considerarse que si bien los estudios preliminares son importantes, estos deben contar con una base conceptual y empírica que sustente sus los análisis estadísticos empleados. Por ejemplo, en el caso de estudios que pongan a prueba algún modelo de la IE, partir de un análisis estructural exploratorio es cuestionable puesto que se tiene una teoría de base (Ferrando \& Anguiano-Carrasco, 2010). Asimismo, el uso de algunos coeficientes de confiabilidad está restringido por el cumplimiento de supuestos estadísticos (e.g., coeficiente $\alpha$ ), y cuando estos no se cumplen, es recomendable contar con alternativas que puedan brindar un panorama más adecuado sobre la confiabilidad.

Entre las limitaciones del presente estudio, se destacan las características de la muestra $(n<500$, predominantemente mujeres, y la mayoría adultos jóvenes, universitarios), que pudieron sesgar los resultados, ante lo cual es recomendable ampliar la muestra a diferentes edades y homogeneizarla según el sexo. Posteriormente, es necesario realizar estudios de invarianza de medición según el sexo y la edad, ya que existe evidencia de que la edad afecta de forma diferencial la percepción de la IE (Bar-On, 2000); y en cuanto al sexo, al evaluar aspectos vinculados con las emociones y la personalidad, es probable que existan diferencias al respecto (Hyde, 2014; Zell, Krizan, \& Teeter, 2015). Del mismo modo, el carácter intencional del muestreo limita la generalización de resultados. Por otro lado, la replicabilidad del error correlacionado modelado debe ser evaluada intencionalmente en otra muestra de participantes. Finalmente, debe considerarse que el presente estudio no es conclusivo, pero los hallazgos muestran el aporte en cuanto a la consecución de una medida breve, y en español, para la evaluación de la IE desde el enfoque mixto.

\section{Referencias}

Abanto, Z., Higueras, L., \& Cueto, J. (2000). ICE Inventario de Cociente Emocional de Bar-On. Lima-Perú.

Abdollahi, A., \& Abu-Talib, M. (2016). Selfesteem, body-esteem, emotional intelligence, and social anxiety in a college sample: The moderating role of weight. Psychology, Health \& Medicine, 21(2), 221-225.

doi: 10.1080/13548506.2015.1017825 
Bar-On, R. (1997). The Emotional Quotient Inventory (EQ-i): A test of emotional intelligence. Toronto: Multi-Health Systems.

Bar-On, R. (2000). Emotional and social intelligence: insights from the emotional quotient inventory (EQ-I). In R. Bar-On \& J. Parker, The Handbook of Emotional Intelligence (pp. 363 - 388). San Francisco: Jossey-Bass.

Bar-On, R. (2005). The impact of emotional intelligence on subjective well-being. Perspectives in Education, 23, 41-61.

Bar-On, R. (2006). The Bar-On Model of emotional-social intelligence (ESI). Psicothema, 18(1), 13-25.

Bar-On, R., \& Parker, J. D. A. (2000). Emotional Quotient Inventory: Youth versión (EQ-i: $Y V)$ : Technical manual. Toronto: Multi-Health Systems.

Beaducel, A., \& Herzberg, P. Y. (2006). On the performance of maximum likelihood versus means and variance adjusted weighted least squares estimation in CFA. Structural Equation Modeling, 13, 186-203. doi: 10.1207/s15328007sem1302_2

Bentler, P. M. \& Wu, E. J. C. (2012). EQS 6.2 for windows [Statistical Program]. Encino, CA: Multivariate Software, Inc.

Brackett, M. A., \& Mayer, J. D. (2003). Convergent, discriminant, and incremental validity of competing measures of emotional intelligence. Personality and Social Psychology Bulletin, 29(9), 1147-1158. doi:10.1177/0146167203254596

Brown, T. (2006). Confirmatory factor analysis for applied research. New York: The Guilford Press.

Canivez, G. L. (2016). Bifactor modeling in construct validation of multifactored tests: Implications for understanding multidimensional constructs and test interpretation. In K. Schweizer \& C. DiStefano (Eds., under contract), Principles and methods of test construction: Standards and recent advancements (pp. 247-271). Gottingen, Germany: Hogrefe Publishing.

Cazan, A-M., \& Năstasă, L.E. (2015). Emotional intelligence, satisfaction with life and burnout among university students. Procedia-Social and Behavioral Sciences, 180, 1574-1578. doi:10.1016/j.sbspro.2015.02.309

Cheung, G. W., \& Rensvold, R. B. (2002). Evaluating goodness-of-fit indexes for testing measurement invariance. Structural Equation Modeling, 9(2), 233-255. doi: 10.1207/S15328007SEM0902_5

Cheung, C. K., Cheung, H. Y., \& Hue, M. T. (2015). Emotional intelligence as a basis for self-esteem in young adults. The Journal of Psychology, 149(1), 63-84. doi:10.1080/00223980.2013.838540

Clark, L. A., \& Watson, D. (1995). Constructing Validity: Basic issues in Objective Scale Development. Psychological Assessment, 7(3), 309-319. doi:10.1037/1040-3590.7.3.309

Cronbach L. J. (1951). Coefficient alpha and the internal structure of tests. Psychometrika, 16(3), 297-334. doi:10.1007/BF02310555

Dawda, D., \& Hart, S.D. (2000). Assessing emotional intelligence: Reliability and validity of the Bar-On Emotional Quotient Inventory (EQ-i) in university students. Personality and Individual Differences, 28, 797-812. doi:10.1016/S0191-8869(99)00139-7

Dimitriu, O., \& Negrescu, M., (2015). Emotional intelligence and the tendency to use dysfunctional cognitive schemas. ProcediaSocial and Behavioral Sciences, 187, 301306. doi:10.1016/j.sbspro.2015.03.056

Dominguez-Lara, S. (en prensa). Ítem único de ansiedad ante exámenes: Evidencias de validez convergente e incremental en estudiantes universitarios. Educación Médica. doi:10.1016/j.edumed.2017.04.004

Dominguez-Lara, S. (2012). Propuesta para el cálculo del Alfa Ordinal y Theta de Armor. Revista de Investigación en Psicología, 15(1), 213-217.

Dominguez-Lara, S. (2014). ¿Matrices Policóricas/Tetracóricas o Matrices Pearson? Un estudio metodológico. Revista Argentina de Ciencias del Comportamiento, 6(1), 39-48.

Dominguez-Lara, S. (2016). Evaluación de la confiabilidad del constructo mediante el Coeficiente $\mathrm{H}$ : breve revisión conceptual y aplicaciones. Psychologia: Avances en la disciplina, $\quad$ 10(2), 87-94. doi:10.21500/19002386.2134 
Dominguez-Lara, S, \& Merino-Soto, C. (en prensa). Versión breve de la Escala de Afrontamiento ante la Ansiedad e Incertidumbre Pre-examen (COPEAU) en universitarios peruanos. Educación Médica. doi: 10.1016/j.edumed.2017.04.011

Dominguez-Lara, S., \& Merino-Soto, C. (2015a). Una versión breve del Cognitive Emotional Regulation Questionnaire: Análisis estructural del CERQ-18 en estudiantes universitarios limeños. Revista Peruana de Psicología y Trabajo Social, 4(1), 25-36.

Dominguez-Lara, S. \& Merino-Soto, C. (2015b) ¿Por qué es importante reportar los intervalos de confianza del coeficiente alfa de Cronbach? Revista Latinoamericana de Ciencias Sociales, Niñez y Juventud, 13(2), 1326-1328.

Dominguez-Lara, S., Merino-Soto, C., \& Navarro-Loli, J. (2016). Estimación de la Confiabilidad en Mediciones de dos ítems: el Coeficiente Angoff-Feldt. Revista Digital de Investigación en Docencia Universitaria, 10(1), 34-40. doi:10.19083/ridu.10.463

Dunn, T. J., Baguley, T., \& Brunsden, V. (2014). From alpha to omega: A practical solution to the pervasive problem of internal consistency estimation. British Journal of Psychology, 105(3), 399-412. doi:10.1111/bjop.12046

Extremera, N., \& Fernández-Berrocal, P. (2004). EL uso de las medidas de habilidad en el ámbito de la inteligencia emocional. Ventajas e inconvenientes con respecto a las medidas de autoinforme. Boletín de Psicología, 80, 5977.

Extremera, N., Fernández-Berrocal, P., Mestre, J., \& Guil, R. (2004). Medidas de evaluación de la inteligencia emocional. Revista Latinoamericana de Psicología, 36(2), 209228.

Feldt, L. S., \& Charter, R. A. (2003). Estimating the reliability of a test Split into two parts of equal or unequal length. Psychological Methods, 8(1), 102-109. doi:10.1037/1082989X.8.1.102

Ferrándiz, C., Hernández, D., Bermejo, R., Ferrando, M., \& Sáinz, M. (2012). La inteligencia emocional y social en la niñez y adolescencia: validación castellana de un instrumento para su medida. Revista de Psicodidáctica, 17(2), 309-339.

doi:10.1387/Rev.Psicodidact.2814

Ferrando, P.J., \& Anguiano-Carrasco, C. (2010). El análisis factorial como técnica de investigación en psicología. Papeles del Psicólogo, 31(1), 18-33.

Fleming, J., \& Merino, C. (2005). Medidas de simplicidad y ajuste factorial: Un enfoque para la construcción y revisión de escalas derivadas factorialmente. Revista de Psicología, 23(2), 252-266.

Gadermann, A. M., Guhn, M., \& Zumbo, B.D. (2012). Estimating ordinal reliability for Likert-type and ordinal item response data: A conceptual, empirical, and practical guide. Practical Assessment, Research, \& Evaluation, 17(3). Recuperado de: http://pareonline.net/getvn.asp?v=17\&n=3.

Garaigordobil, M., \& Peña, A. (2014). Intervención en las habilidades sociales: efectos en la inteligencia emocional y la conducta social. Psicología Conductual, 22(3), 551-567.

Graham, J. M., Guthrie, A. C., \& Thompson, B. (2003). Consequences of not interpreting structure coefficients in published CFA research: A reminder. Structural Equation Modeling, 10(1), 142-153. doi:10.1207/S15328007SEM1001_7

Hancock, G. R., \& Mueller, R. O. (2001). Rethinking construct reliability within latent variable systems. En R. Cudeck, S. H. C. du Toit \& D. Sörbom (Eds.), Structural equation modeling: Past and present. A Festschrift in honor of Karl G. Jöreskog (pp. 195-261). Chicago: Scientific Software International.

Hemmati, T., Mills, J. F., \& Kroner, D. G. (2004). The validity of the Bar-On emotional intelligence quotient in an offender population. Personality and Individual Differences, 37, 695-706. doi:10.1016/j.paid.2003.10.003

Hyde, J. S. (2014). Gender similarities and differences. Anual Review of Psychology, 65, 373-398. doi:10.1146/annurev-psych-010213-115057

Inzunza, B., Ortiz, L., Pérez, C., Torres, G., McColl, P., Meyer, A., Matus, O., Bastías, N., $\&$ Bustamante, C. (2015). Estructura factorial y confiabilidad del cuestionario de 
satisfacción académica en estudiantes de medicina chilenos. Revista Iberoamericana de Diagnóstico y Evaluación - e Avaliação Psicológica, 40(2), 73-82.

Joseph, D. L., Jin, J., Newman, D. A., \& O’Boyle, E. H. (2015). Why does self-reported emotional intelligence predict job performance? A meta-analytic investigation of mixed EI. Journal of Applied Psychology, 100(2), 298-342. doi:10.1037/a0037681

Kaiser, H. (1960). The application of electronic computers to factor analysis. Educational and Psychological Measurement, 20, 141-151. doi:10.1177/001316446002000116

Kroenke, K., Spitzer, R., \& Williams, J. (2003). The Patient Health Questionnarie-2: Validity of Two-item Depression Screener. Medical Care, 41(11), 1284-1292. doi:10.1097/01.MLR.0000093487.78664.3C

Kroenke, K., Spitzer, R., Williams, J., Monahan, P. \& Lowe, B. (2007). Anxiety disorders in primary care: Prevalence, impairment, comorbidity, and detection. Annals of International Medicine, 146, 317-325. doi:10.7326/0003-4819-146-5-200703060-00004

Lee, S.-Y., Poon, W.-Y., \& Bentler, P. M. (1995). A two-stage estimation of structural equation models with continuous and polytomous variables. British Journal of Mathematical and Statistical Psychology, 48(2), 339-358. doi: 10.1111/j.2044-8317.1995.tb01067.x

López-Zafra, E., Pulido, M., \& Berríos, P. (2014). EQI-versión corta (EQI-C). Adaptación y validación al español del EQ-i en universitarios. Boletín de Psicología, 110, 2136.

Lloyd, S. J., Malek-Ahmdi, M., Barclay, K., Fernandez, M. R., \& Chartrand, M. S. (2012). Emotional Intelligence (EI) as a predictor of depression status in older adults. Archives of Gerontology and Geriatrics, 55, 570-573. doi:10.1016/j.archger.2012.06.004

Lloret-Segura, S., Ferreres-Traver, A., HernándezBaeza, A., \& Tomás-Marco, I. (2014). El análisis factorial exploratorio de los ítems: Una guía práctica, revisada y actualizada. Anales de Psicología, 30(3), 1151-1169. doi:10.6018/analesps.30.3.199361

Malgady, R. (2007). How skew are psychological data? A standardized index of effect size. The
Journal of General Psychology, 134(3), 355359. doi:10.3200/GENP.134.3.355-360

Martin-Raugh, M. P., Kell, H. J., \& Motowidlo, S. J. (2016). Prosocial knowledge mediates effects of agreeableness and emotional intelligence on prosocial behavior. Personality and Individual Differences, 90, 41-49. doi:10.1016/j.paid.2015.10.024

Mayer, J. D., \& Salovey, P. (1997). What is emotional intelligence? En P. Salovey y D. Sluyter (Eds.). Emotional development and emotional intelligence: Implications for educators (pp 3-31). New York: Basic Books.

Mayer, J.D., Salovey, P., \& Caruso, D. (2002). Mayer-Salovey-Caruso Emotional Intelligence Test (MSCEIT) user's manual. Toronto: Multi-Health Systems.

McDonald R.P. (1999). Test theory: A unified treatment. Mahwah, N.J.: L. Erlbaum Associates.

Medrano, L., Moretti, L., \& Ortiz, A. (2015). Medición del Engagement Académico en Estudiantes Universitarios. Revista Iberoamericana de Diagnóstico y Evaluación - e Avaliação Psicológica, 40(2), 114-123.

Merino-Soto, C., Dominguez-Lara, S., \& Fernandez-Arata, M. (2017). Validación inicial de una Escala Breve de Satisfacción con los Estudios (EBSE) en estudiantes universitarios de Lima. Educación Médica, 18(1), 74-77. doi: 10.1016/j.edumed.2016.06.016

Merino C., Navarro J., \& García W. (2014). Revisión de la consistencia interna del Inventario de Inteligencia Emocional de BarOn, EQ-I: YV. Revista Peruana de Psicología y Trabajo Social, 3(1),141-154.

Meyer, J. P. (2010). Reliability. New York: Oxford University Press.

Mikolajczak, M., \& Van Bellegem, S. (2017). Increasing emotional intelligence to decrease healthcare expenditures: How profitable would it be? Personality and Individual Differences, 116, 343-347. doi:10.1016/j.paid.2017.05.014

Mshellia, P. S., Malachy, D. Y., Sabo, B., \& AbuAbdissamad, A. M. (2016). Emotional intelligence and contextual performance among nursing professionals in Jos Metropolis, Plateau State, Nigeria. Journal of 
Good Governance and Sustainable Development in Africa, 3(1), 19-33.

Năstasă, L. E., \& Fărcaş, A. D. (2015). The effect of emotional intelligence on burnout in healthcare professionals. Procedia-Social and Behavioral Sciences, 187, 78-82. doi: 10.1016/j.sbspro.2015.03.015

Navarro, J., Merino, C., Dominguez, S., \& Fleming, J. (2016). Importancia de la evaluación de la simplicidad factorial: Reanálisis a Zicado, Palma y Garrido (2012). Revista Latinoamericana de Ciencias Sociales, Niñez y Juventud, 1682-1684.

Newsome, S., Day, A. L., \& Catano, V. M. (2000). Assessing the predictive validity of emotional intelligence. Personality and Individual Differences, 29, 1005-1016. doi:10.1016/S0191-8869(99)00250-0

Nolidin, K., Downey, L. A., Hansen, K., Schweitzer, I., \& Stough, C. (2013). Associations between social anxiety and emotional intelligence within clinically depressed patients. Psychiatric Quarterly, 84(4), 513-521.

doi:10.1007/s11126-013-9263-5

Páez, M., \& Castaño, J. (2015). Inteligencia emocional y rendimiento académico en estudiantes universitarios. Psicología desde el Caribe, 32(2), 268-285.

Parker, J. D., Keefer, K. V., \& Wood, L. M. (2011). Toward a brief multidimensional assessment of emotional intelligence: psychometric properties of the Emotional Quotient Inventory-Short Form. Psychological Assessment, 23(3), 762-777. doi:10.1037/a0023289.

Parker, J. D., Summerfeldt, L. J., Taylor, R. N., Kloosterman, P. H., \& Keefer, K. V. (2013). Problem gambling, gaming and Internet use in adolescents: Relationships with emotional intelligence in clinical a special needs samples. Personality and Individual Differences, 55, 288-293. doi:10.1016/j.paid.2013.02.025

Pascual-Ferrá. P, \& Beatty, M. J. (2015). Correcting internal consistency estimates inflated by correlated item errors. Communication Research Reports, 32(4): 347352. doi: 10.1080/08824096.2015.1089858
Pérez-Fuentes, M., Gázquez, J., Mercader, I., \& Molero M. (2014). Brief Emotional Intelligence Inventory for Senior Citizens (EQ-i-M20). Psicothema, 26(4), 524-530. doi:10.7334/psicothema2014.166

Petrides, K.V., \& Furnham, A. (2006). The role of trait emotional intelligence in a genderspecific model of organizational variables. Journal of Applied Social Psychology, 36(2), 552-569. doi:10.1111/j.0021-9029.2006.00019.x

Petrides, K. V., Mikolajczak, M., Mavroveli, S., Sanchez-Ruiz, M. J., Furnham, A., \& PérezGonzález, J. C. (2016). Developments in trait emotional intelligence research. Emotion Review, 8(4), 335-341. doi:10.1177/1754073916650493

Pishghadam, R., \& Sahebjam, S. (2012). Personality and emotional intelligence in teacher burnout. The Spanish Journal of Psychology, 15(1), 227-236. doi:10.5209/rev_SJOP.2012.v15.n1.37314

Raykov, T. (2001). Bias in coefficient alpha for fixed congeneric measures with correlated errors. Applied Psychological Measurement, 25(1), 69-76. doi: 10.1177/01466216010251005

Raykov, T., \& Hancock, G. R. (2005). Examining change in maximal reliability for multiplecomponent measuring instruments. British Journal of Mathematical and Statistical Psychology, 58(1), 65-82. doi:10.1348/000711005X38753

Reise, S. P. (2012). The rediscovery of bifactor measurement models. Multivariate Behavioral Research, 47(5), 667-696. doi:10.1080/00273171.2012.715555

Reise, S. P., Scheines, R., Widaman, K. F., \& Haviland, M. G. (2013). Multidimensionality and structural coefficient bias in structural equation modeling: A bifactor perspective. Educational and Psychological Measurement, 73(1), 5-26. doi:10.1177/0013164412449831

Reise, S. P., Waller, N. G., \& Comrey, A. L. (2000). Factor analysis and scale revision. Psychological Assesment, 12, 287-297. doi:10.1037/1040-3590.12.3.287

Rios, J., \& Wells, C. (2014). Validity evidence based on internal structure. Psicothema, 26(1), 108-116. doi:10.7334/psicothema2013.260

Robins, R. W., Tracy, J. L., Trzesniewski, K. H., Potter, J., \& Gosling, S. D. (2001). Personality 
correlates of self-Esteem. Journal of Research in Personality, 35, 463-482.

doi:10.1006/jrpe.2001.2324

Robins, R. W., Trzesniewski, K. H., Tracy, J. L., Gosling, S. D., \& Potter, J. (2002). Global self-esteem across the life-span. Psychology and Aging, 17, 423-434. doi:10.1037/08827974.17.3.423

Robins, R., Hendin, H., \& Trzeaniewski, K. (2001). Measuring global self-esteem: Construct validation of a single-item measure and the Rosenberg Self-Esteem Scale. Personality and Social Psychology Bulletin, 27, 151-161. doi:10.1177/0146167201272002

Rodriguez, A., Reise, S. P., \& Haviland, M. G. (2016). Evaluating bifactor models: Calculating and interpreting statistical indices. Psychological Methods, 21(2), 137-150. doi:10.1037/met0000045

Sáinz, M., Ferrándiz, C., Fernández, C., \& Ferrando, M. (2014). Propiedades psicométricas del Inventario de Cociente Emocional EQ-i:YV en alumnos superdotados y talentosos. Revista de Investigación Educativa, 32 (1), 41-55. doi:10.6018/rie.32.1.162501

Salovey, P., \& Mayer, J. D. (1990). Emotional intelligence. Imagination, Cognition, and Personality, 9, 185-211.

Salguero, J. M., Extremera, N., \& FernándezBerrocal, P. (2012). Emotional intelligence and depression: The moderator role of gender. Personality and Individual Differences, 53(1), 29-32. doi:10.1016/j.paid.2012.02.006

Sánchez-Álvarez, N., Extremera, N., \& Fernández-Berrocal, P. (2016). The relation between emotional intelligence and subjective well-being: A meta-analytic investigation. The Journal of Positive Psychology, 11(3), 276285. doi:10.1080/17439760.2015.1058968

Satorra, A., \& Bentler, P. M. (1994). Corrections to test statistics and standard errors in covariance structure analysis. In A. von Eye \& C. C. Clogg (Eds.), Latent variables analysis: Applications for developmental research (pp. 399-419). Thousand Oaks, CA: Sage.

Siegling, A. B, Saklofske, D. H., Vesely, A. K., \& Nordstokke, D. W. (2012). Relations of emotional intelligence with gender-linked personality: Implications for a refinement of EI constructs. Personality and Individual Differences, 52(7), 776-781. doi:10.1016/j.paid.2012.01.003

Schokman, C., Downey, L. A., Lomas, J., Wellham, D., Wheaton, A., Simmons, N., \& Stough, C. (2014). Emotional intelligence, victimization, bullying behaviours and attitudes. Learning and Individual Differences, 36, 194-200. doi:10.1016/j.lindif.2014.10.013

Sörbom, D. (1989). Model modification. Psychometrika, 54(3), 371-384. doi:10.1007/BF0229623

Stanimirovic, R., \& Hanrahan, S. (2012). Examining the dimensional structure and factorial validity of the Bar-On Emotional Quotient Inventory in a simple of male athletes. Psychology of Sport and Exercise, 13, 44-50. doi:10.1016/j.psychsport.2011.07.009

Suárez, Y. P., Restrepo, D. E., \& Caballero, C. (2016). Suicidal ideation and its relationship to emotional intelligence in Colombian college students. Revista de la Universidad Industrial de Santander. Salud, 48(4), 470478. doi: 10.18273/revsal.v48n4-2016005

Terwee, C. B., Bot, S. D. M., de Boer, M. R., van der Windt, D. A. W. M., Knol, D. L., Dekker, J., Bouter, L. M., \& de Vet, H. C. W. (2007). Quality criteria wereproposed for measurement properties of health status questionnaires. Journal of Clinical Epidemiology, 60(1), 34-42. doi:10.1016/j.jclinepi.2006.03.012

Thompson, B. (1997). The importance of structure coefficients in structural equation modeling confirmatory factor analysis. Educational and Psychological Measurement, 57(1), 5-19. doi:10.1177/0013164497057001001

Torres, P., \& Cobo, J. (2017). Estrategias de gestión de la inteligencia emocional para la prevención del Síndrome de Burnout en docentes de aula. Educ@ción en Contexto, 2(4), 281-295.

Ugarriza, N. (2001). La evaluación de la Inteligencia Emocional a través de inventario de Bar-On (I-CE) en una muestra de Lima metropolitana. Persona, 4, 129-160.

Ugarriza, N., \& Pajares, L. (2005). La evaluación de la inteligencia emocional a través del 
inventario de Bar-On ICE-NA, en una muestra de niños y adolescentes. Persona, 8, 11-58.

Webb, C. A., Schwab, Z. J., Weber, M., DelDonno, S., Kipman, M., Weiner, M. R., \& Killgore, W. D. S. (2013). Convergent and divergent validity of integrative versus mixed model measures of emotional intelligence. Intelligence, 41, 149-156.

Zell, E., Krizan, Z., \& Teeter, S. R. (2015). Evaluating gender similarities and differences using metasynthesis. American Psychologist, 70(1), 10-20. doi:10.1037/a0038208.

Zinbarg, R. E., Yovel, I., Revelle, W., \& McDonald, R. P. (2006). Estimating generalizability to a latent variable common to all of a scale's indicators: A comparison of estimators for $\omega \mathrm{h}$. Applied Psychological Measurement, 30(2), 121-144.

doi:10.1177/0146621605278814 